\title{
Incidence of Hematopoietic Sarcoma in Iranian Population
}

\author{
Maryam Mabani, MD; Mohammad Esmaeil Akbari, MD²; Hamid Rezvani, MD; Hamide Rahmani, MD; Atieh Akbari, MD²; \\ Alireza Raeisi, $\mathrm{MD}^{3}$; Ghasem Janbabaee, $\mathrm{MD}^{4}$; Davood Bashash, $\mathrm{PhD}^{5}$; Sina Salari, $\mathrm{MD}^{6 *}$ \\ 'Department of Hematology and Oncology, Taleghani Hospital, Shahid Beheshti University of Medical Sciences, Tehran, Iran \\ ${ }^{2}$ Cancer Research Center, Shahid Beheshti University of Medical Sciences, Tehran, Iran \\ ${ }^{3}$ Department of Internal Medicine, Shiraz University of Medical Science, Shiraz, Iran \\ ${ }^{4}$ Gastrointestinal Cancer Research Center, Faculty of Medicine, Mazandaran University of Medical Science, Sari, Iran \\ ${ }^{5}$ Department of Hematology and Blood Banking, School of Allied Medical sciences, Shahid Beheshti University of Medical Sciences, \\ Tehran, Iran \\ ${ }^{6}$ Department of Medical Oncology, Hematology and Bone Marrow Transplantation, Taleghani Hospital, Shahid Beheshti University \\ of Medical Sciences, Tehran, Iran
}

\begin{abstract}
Background: The heterogeneous nature of hematopoietic sarcoma has restricted the diagnosis and treatment of this disease to the extent that annually, several patients lose their lives. Given the lack of comprehensive epidemiologic information on the incidence of hematopoietic sarcoma in the Iranian population, we designed the present study to evaluate the distribution pattern of this disease.

Methods: In this national population-based cancer registry study, we collected data from patients diagnosed with hematopoietic sarcoma who were registered in the Iran National Cancer Registry (INCR) between 2009 and 2013. For each patient, the variables of age, sex, province, year of diagnosis, site of involvement and morphology were collected.

Results: In 45 cases from 18 provinces of Iran, we found that the incidence rate of the disease was $0.60(95 \% \mathrm{Cl}: 0.44-0.80)$ per million persons. Among all provinces, Ilam had the highest incidence of hematopoietic sarcoma with a rate of $2(95 \% \mathrm{Cl}: 0.05-$ 11.14) per million persons, while Isfahan had the lowest incidence with a rate of 0.21 (95\% Cl: $0.01-1.16)$ per million persons. The incidence rate of the disease increased with age and the disease was slightly more common in men $(0.63$ [95\% $\mathrm{Cl}$ : $0.41-0.94]$ vs. 0.56 [95\% Cl: 0.35-0.86] per million persons). The frequency of hematopoietic sarcoma in connective and soft tissues was higher than other anatomical sites and we found that myeloid morphology was the most prevalent morphology.

Conclusion: The resulting data provided a valuable perspective on the distribution pattern of hematopoietic sarcoma in Iran; however, further studies are required to confirm these results.

Keywords: Hematopoietic System, Incidence, Iran, Sarcoma

Cite this article as: Mabani M, Akbari ME, Rezvani H, Rahmani H, Akbari A, Raeisi A, et al. Incidence of hematopoietic sarcoma in iranian population. Arch Iran Med. 2021;24(6):461-466. doi: 10.34172/aim.2021.66
\end{abstract}

Received: December 2, 2020, Accepted: March 13, 2021, ePublished: June 1, 2021

\section{Introduction}

Hematopoietic sarcoma is a broad family of neoplastic diseases of immature blood cells, which could affect different connective tissues and display varied morphology. Notably, the first type of this malignancy that attracts attention is myeloid sarcoma (MS), which is an extramedullary manifestation of neoplastic myeloid cells with the ability to disrupt normal tissue structure. ${ }^{1,2}$ MS may occur with acute myeloid leukemia (AML) before or after a relapse and is less associated with myeloproliferative neoplasm or myelodysplastic disorder. ${ }^{3,4}$ The incidence of isolated MS in adults is $2 \%$. The patients' age at the onset of disease varies widely, ranging from the age of 1 to 81 years. ${ }^{5}$ This malignancy often occurs in soft tissues, bones, peritoneum, lymph nodes, and the gastrointestinal tract. ${ }^{3}$

Given the heterogeneous characteristics of hematopoietic sarcoma, in particular MS, the diagnosis of primary sarcoma and its treatment have turned into one of the most challenging issues to the extent that annually, several patients lose their lives due to either misdiagnosis or treatment failure. The misdiagnosis rate for this disease has been reported to be $75 \%$ and most of the detected cases were diagnosed as large cell lymphoma followed by malignant lymphoproliferative disorders. ${ }^{6,7}$ Another challenge hindering successful management of the disease is the lack of an appropriate treatment protocol. Surgery has been proposed to be the only treatment strategy for this malignancy; however, a considerable number of patients experience disease recurrence or eventually progress to $\mathrm{AM},{ }^{8,9}$ suggesting that hematopoietic sarcoma is a fatal malignancy with a poor prognosis. ${ }^{12}$ Despite the dismal survival of patients, the success of bone marrow transplantation (BMT) and allogeneic stem cell transplantation in the treatment of $17 \%$ of patients has shifted the paradigm of treatment protocol. ${ }^{10}$ Along with the prosperity of BMT, a new 
stream of efforts has been dedicated to finding proper targeted therapies for patients with MS in a hope to alter treatment choices and disease prognosis. ${ }^{11}$ However, these efforts may not develop into a valuable therapeutic approach, unless thorough knowledge has been obtained about the epidemiology of the disease. Given this and considering the lack of comprehensive information on the incidence of hematopoietic sarcoma in Iran, we aimed to evaluate the distribution pattern of the disease according to different variables of age, sex, province of residence, year of diagnosis, site of involvement and morphology.

\section{Patients and Methods}

\section{Data Collection}

In this national population-based cancer registry study, we studied the medical records of all patients with a pathologic diagnosis of hematopoietic sarcoma registered in the Iran National Cancer Registry (INCR) between 2009 and 2013. For each patient in the data collection form, the variables of age, sex, province of residence, year of diagnosis, site of involvement based on clinical and pathologic findings and morphology of hematopoietic sarcoma based on pathology report were collected.

\section{Statistical Analysis}

All statistical analyses were performed using R-CRAN software version 4.0.3. The studied categorical variables were described using frequency and percentage. Incidence rate is the number of new cases of hematopoietic sarcoma during the time interval between 2009 and 2013 divided by summed person-years of observation or average population during this time interval. Cancer registries are involved in calculating incidence rates for entire populations and the denominator for these rates cannot be derived from a knowledge of each individual's contribution to the population at risk. This is, therefore, generally approximated by the mid-year population (or the average of the population at the beginning and end of the year or 5-year period), which is obtained from a census department.

The incidence rate of hematopoietic sarcoma in a 5-year period (2009 to 2013) with the exact 95\% confidence interval was reported by each province, age category and other variables. Moreover, age standardized incidence rate (ASR) was reported using the World Health Organization (WHO) standard population.

\section{Results}

The incidence rate of hematopoietic sarcoma in the Iranian population was reported by provinces, age groups, and gender. The crude incidence rate and the ASR were estimated to be 0.60 (95\% CI: $0.44-0.80)$ and 0.68 (95\% CI: 0.49-0.93) per million persons, respectively.

Distribution and the Incidence of Hematopoietic Sarcoma in Different Provinces of Iran

In total, over a 5-year period (2009 to 2013), 45 cases with pathologic diagnosis of hematopoietic sarcoma from 31 provinces of Iran were registered in the INCR. Among all provinces, the highest incidence was reported in Ilam with the rate of 2 (95\% CI: 0.05-11.14) per million persons and the lowest incidence was reported in Isfahan with the rate of 0.21 (95\% CI: 0.01-1.16) per million persons (Figure 1).

To evaluate the incidence rate of hematopoietic sarcoma in different provinces of Iran, we also calculated the crude incidence rate of the malignancy, which is estimated to be 0.60 (95\% CI: $0.44-0.80)$ per million persons in the indicated time period (Table 1 ).

\section{Evaluating the Distribution Pattern of Hematopoietic Sarcomas in Different Age Groups and Genders}

Age has been considered as a valuable parameter in determining the characteristic of human cancers and hematopoietic sarcomas is not an exception. Our results showed that hematopoietic sarcomas had different peaks in different age groups. We also found that the majority of patients diagnosed with hematopoietic sarcoma were either young with the average age of 30-34 years (n $=8,19 \%)$ or in the range of $70-74$ years $(n=5,11 \%)$. While this malignancy had the lowest incidence rate in children in the age range of $10-14$ years $(0.00$ [95\% CI: $0.00-0.53$ ] per million persons), its incidence rate in the aforementioned age groups was 1.16 (95\% CI: 0.50-2.28) per million persons and 4.55 (95\% CI: $1.48-10.61)$ per million persons, respectively (Table 2).

Table 1. Incidence Rate of Hematopoietic Sarcoma by Patients' Province of the Residence of Patients

\begin{tabular}{|c|c|c|c|}
\hline Province & $\begin{array}{l}\text { Population } \\
\left(\times 10^{6}\right)\end{array}$ & $\begin{array}{l}\text { Number } \\
\text { of Cases } \\
(n=45)\end{array}$ & $\begin{array}{c}\text { Incidence Rate } \\
(95 \% \mathrm{Cl}) \\
\text { (per } 1 \text { million } \\
\text { persons) }\end{array}$ \\
\hline Tehran & 12 & 10 & $0.83(0.40,1.53)$ \\
\hline $\begin{array}{l}\text { Khorasan } \\
\text { Razavi }\end{array}$ & 6 & 3 & $0.50(0.10,1.46)$ \\
\hline Isfahan & 4.8 & 1 & $0.21(0.01,1.16)$ \\
\hline Fars & 4.5 & 4 & $0.89(0.24,2.28)$ \\
\hline East Azerbaijan & 3.7 & 1 & $0.27(0.01,1.51)$ \\
\hline Khoozestan & 4.5 & 1 & $0.22(0.01,1.24)$ \\
\hline Mazandaran & 3 & 5 & $1.67(0.54,3.89)$ \\
\hline Alborz & 2.4 & 2 & $0.83(0.10,3.01)$ \\
\hline Kerman & 2.9 & 4 & $1.38(0.38,3.53)$ \\
\hline Ghazvin & 1.2 & 1 & $0.83(0.02,4.64)$ \\
\hline Ardebil & 1.2 & 1 & $0.83(0.02,4.64)$ \\
\hline South Khorasan & 0.6 & 1 & $1.67(0.04,9.29)$ \\
\hline Golestan & 1.8 & 2 & $1.11(0.13,4.01)$ \\
\hline $\begin{array}{l}\text { West } \\
\text { Azerbaijan }\end{array}$ & 3 & 4 & $1.33(0.36,3.41)$ \\
\hline Lorestan & 1.7 & 1 & $0.59(0.01,3.28)$ \\
\hline Kermanshah & 1.9 & 2 & $1.05(0.13,3.80)$ \\
\hline Ilam & 0.5 & 1 & $2.00(0.05,11.14)$ \\
\hline $\begin{array}{l}\text { Sistan \& } \\
\text { Baloochestan }\end{array}$ & 2.5 & 1 & $0.40(0.01,2.23)$ \\
\hline Other & 16.8 & 0 & $0.00(0.00,0.18)$ \\
\hline Total & 75 & 45 & $0.60(0.44,0.80)$ \\
\hline
\end{tabular}


Apart from age, the incidence of many human cancers may also differ with gender. Given this, it was of particular interest to evaluate if there was a difference in the incidence rate of hematopoietic sarcoma between males and females. Our results showed that the disease has almost a similar distribution according to gender, as among the studied population, 24 cases pertained to males (53\%) and 21 cases were diagnosed in females (47\%) (Table 3). This finding was also confirmed by the calculation of incidence rate, as we found that the incidence rate of the disease was slightly higher in men (0.63 [95\% CI: $0.41-0.94]$ vs. 0.56 [95\% CI: 0.35-0.86] per million persons) (Table 3).

\section{Studying Hematopoietic Sarcoma in Iranian Patients According to the Tissue Origin}

Based on the origin of the affected tissue, hematopoietic sarcoma may have different anatomical distribution and morphology. In the present study, we found that the connective and soft tissue $(n=9,20 \%)$ and the nervous system $(n=6,13 \%)$ were the most common anatomical sites in the diagnosed cases. Moreover, the most common

Table 2. Incidence Rate of Hematopoietic Sarcoma According to Patients' Age Category of the Patients

\begin{tabular}{lccc}
\hline Age Range (y) & $\begin{array}{c}\text { Population } \\
(\times \mathbf{1 0})\end{array}$ & $\begin{array}{c}\text { Number of } \\
\text { Cases } \\
(\mathbf{n}=\mathbf{4 5})\end{array}$ & $\begin{array}{c}\text { Incidence Rate } \\
(\mathbf{9 5} \mathbf{\%} \mathbf{C l}) \\
\text { (per million } \\
\text { persons) }\end{array}$ \\
\hline $0-4$ & 6.2 & 1 & $0.16(0.00,0.90)$ \\
\hline $5-9$ & 5.6 & 2 & $0.36(0.04,1.29)$ \\
\hline $10-14$ & 5.6 & 0 & $0.00(0.00,0.53)$ \\
\hline $15-19$ & 6.6 & 2 & $0.30(0.04,1.09)$ \\
\hline $20-24$ & 8.4 & 4 & $0.48(0.13,1.22)$ \\
\hline $25-29$ & 8.6 & 3 & $0.35(0.07,1.02)$ \\
\hline $30-34$ & 6.9 & 8 & $1.16(0.50,2.28)$ \\
\hline $35-39$ & 5.5 & 1 & $0.18(0.00,1.01)$ \\
\hline $40-44$ & 4.9 & 1 & $0.20(0.01,1.14)$ \\
\hline $45-49$ & 4.0 & 3 & $0.75(0.15,2.19)$ \\
\hline $50-54$ & 3.5 & 2 & $0.57(0.07,2.06)$ \\
\hline $55-59$ & 2.6 & 3 & $1.15(0.24,3.37)$ \\
\hline $60-64$ & 1.8 & 4 & $2.22(0.61,5.69)$ \\
\hline $65-69$ & 1.3 & 3 & $2.31(0.48,6.74)$ \\
\hline $70-74$ & 1.1 & 5 & $4.55(1.48,10.61)$ \\
\hline $75-79$ & 0.9 & 3 & $3.33(0.69,9.74)$ \\
\hline 80 or more & 0.9 & 0 & $0.00(0.00,3.33)$ \\
\hline Total & 75 & 45 & $0.60(0.44,0.80)$ \\
\hline
\end{tabular}

Table 3. Incidence Rate of Hematopoietic Sarcoma According to Gender

\begin{tabular}{lccc}
\hline Gender & $\begin{array}{c}\text { Population } \\
\left(\times \mathbf{1 0}^{\mathbf{6}}\right)\end{array}$ & $\begin{array}{c}\text { Number of } \\
\text { Cases } \\
(\mathbf{n}=\mathbf{4 5})\end{array}$ & $\begin{array}{c}\text { Incidence Rate } \\
(\mathbf{9 5} \% \mathbf{C l}) \\
(\mathbf{p e r} \mathbf{1} \text { million } \\
\text { persons })\end{array}$ \\
\hline Male & 37.8 & 24 & $0.63(0.41,0.94)$ \\
\hline Female & 37.2 & 21 & $0.56(0.35,0.86)$ \\
\hline Total & 75 & 45 & $0.60(0.44,0.80)$ \\
\hline
\end{tabular}

morphology that was reported for hematopoietic sarcoma in the Iranian population was MS $(\mathrm{n}=30,67 \%)$, which was followed by histiocytic sarcoma ( $\mathrm{n}=7,16 \%)$, mast cell sarcoma ( $\mathrm{n}=5,11 \%)$, Hodgkin's $(\mathrm{n}=2,4 \%)$ and malignant mastocytosis sarcoma $(n=1,2 \%)$ (Figure 2). In agreement with this finding, calculation of incidence rate also showed that the occurrence of MS $(0.40$ [95\% CI $0.27-0.57$ ] per one million persons) was higher than other pathologic morphologies of hematopoietic sarcoma.

\section{Discussion}

The importance of epidemiological studies in cancer is increasing day by day, since the more carefully the cancer incidence is studied, the more obvious it becomes which parameters and drivers may have an influence on its pathogenesis. Hematopoietic sarcoma is one of the challenging malignancies all around the world as it originates from different hematopoietic cells, such as myeloid, mast cell, and lymphoid cells. ${ }^{3,13}$ The heterogeneous characteristics, diverse affected tissues coupled with different clinical manifestations not only have restricted the current knowledge about this malignancy but also made it difficult to diagnose and treat properly. Thus far, the available studies about hematopoietic sarcoma have been presented mainly in case reports. A case of MS following allogeneic stem cell transplantation was introduced by Zhang et al, which was characterized by localized pelvic pain. ${ }^{21}$ In another study, a case of a 47-year-old woman with MS of the vulva was reported who presented with symptoms of AML. ${ }^{22}$ Also, Wang et al described a case of MS of the small bowel, kidney, and mesenteric lymph nodes in a 26 -year-old male patient who suffered from intermittent upper abdominal pain. ${ }^{23}$ In spite of a multitude of case-report studies, still little is known about the epidemiologic features of the disease. Given the rarity and variable incidence of the disease in different geographical areas, we aimed to evaluate the incidence rate as well as the distribution pattern of hematopoietic sarcoma among the Iranian population.

Our results showed that over a 5-year period (20092013), the incidence rate of hematopoietic sarcoma among Iranian population was 0.60 (95\% CI: 0.44-0.80) per million persons, indicating that the incidence of the disease was less than one-third of the rate mentioned in the literature. ${ }^{5,14}$ Misdiagnosis of other cases or failure to properly record or refer suspected patients may, at least partly, explain our results. All the cases were collected from only 18 provinces of Iran and there were no reports from other areas with a population of about 16.8 million. Lack of access to an expert pathologist, adequate facilities and well-equipped centers could also be other reasons why the incidence rate of the disease was lower in Iran compared to other countries. Among the studied provinces, most cases of hematopoietic sarcoma were diagnosed in Tehran and Mazandaran; however, the highest incidence rate for this malignancy pertained to the Ilam province. When it comes to hematopoietic sarcoma, tissue diversity is 

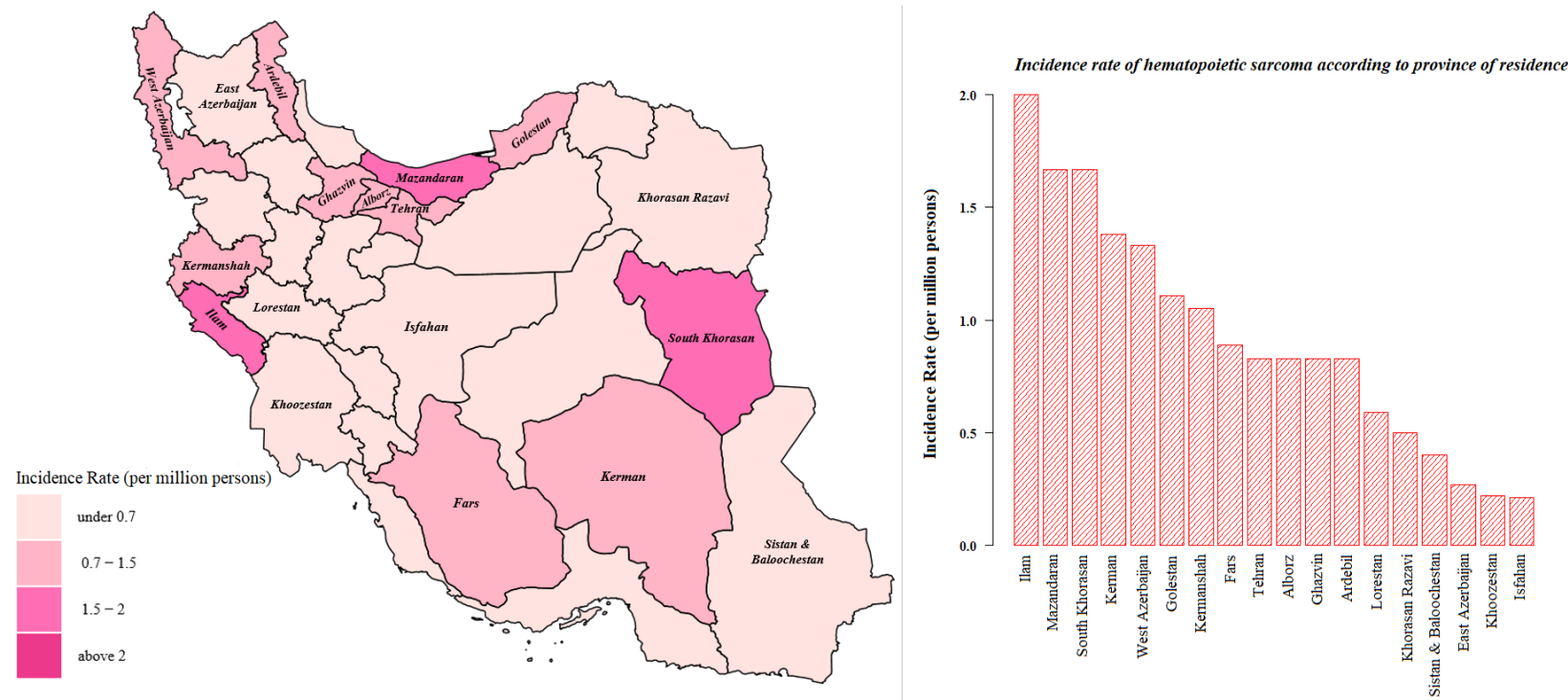

Figure 1. Incidence rate of hematopoietic sarcoma according to province of residence.

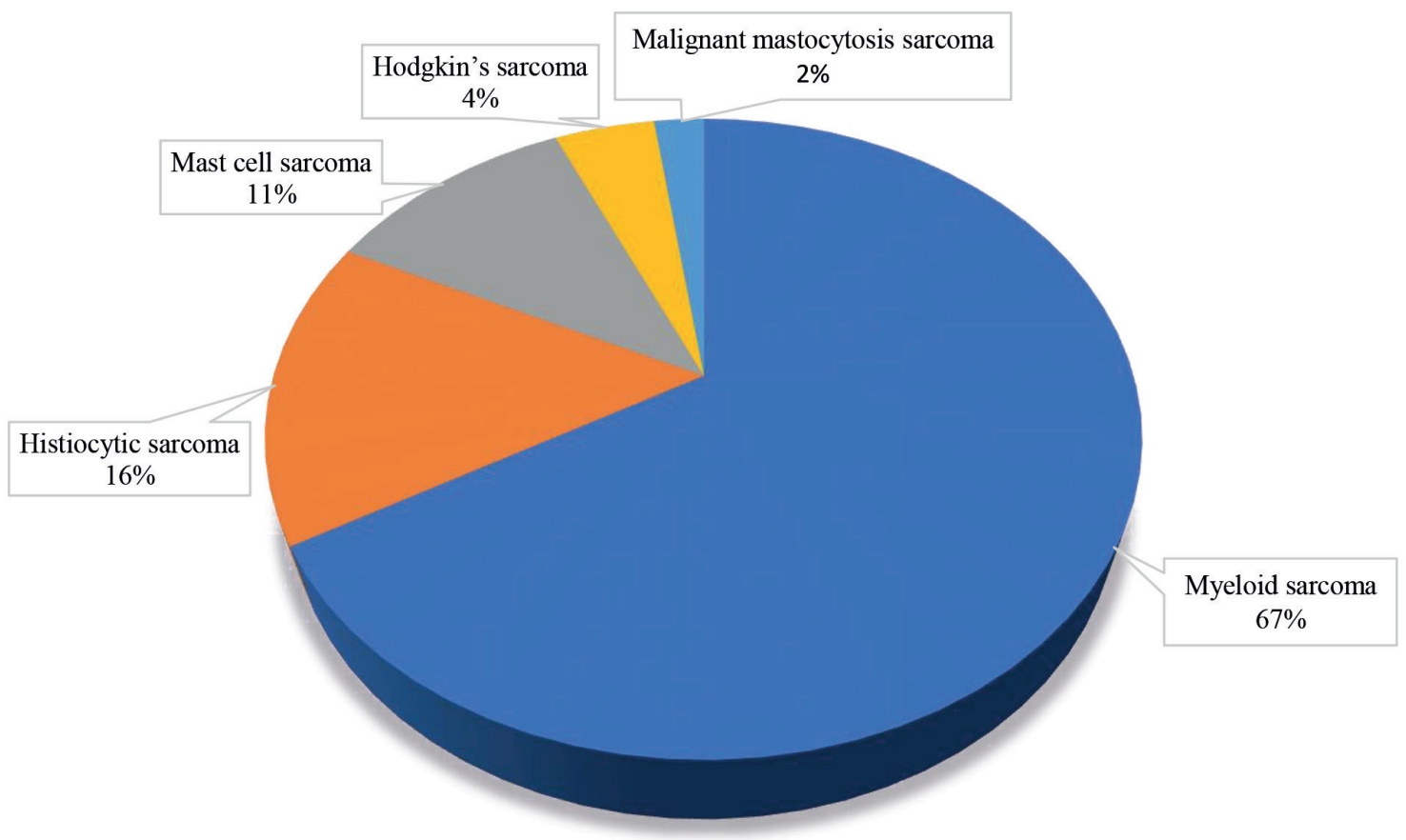

Figure 2. Distribution of Pathologic Morphology in Hematopoietic Sarcoma.

the most challenging issue that has an adverse influence on both diagnosis and treatment approaches. Since the location is diverse, the clinical presentation of the disease will also be various. ${ }^{19}$ It has been established that MS, as the most prevalent type of hematopoietic sarcoma, occurs at any site of the body but the most common locations are soft tissues, bones, peritoneum, lymph node and the gastrointestinal system. Other sites that are presented in the literature are the genitourinary system of males and females and the central nervous system. ${ }^{18}$ Similar to the previous studies, our results indicate that MS is the most common type of hematopoietic sarcoma among the Iranian population. Moreover, we found that hematopoietic sarcoma widely affects connective and soft tissue and the nervous system.

In conclusion, over the last decades, a mounting body of evidence has studied the correlation between the incidences of hematopoietic sarcoma with different parameters. The results of previous studies have shown that although this malignancy occurs at any age in both pediatric and elderly patients, it has the highest prevalence in the pediatric population and $3-5 \%$ of patients with AML may progress to one of the types of hematopoietic sarcomas. Likewise, it has also been reported that the incidence of hematopoietic sarcoma in the adult population is $2 / 1000000 .{ }^{14}$ Of particular interest, our results showed that hematopoietic 
sarcoma was diagnosable in every age group among the Iranian population; however, the incidence of pediatric hematopoietic sarcoma was the lowest among all the age groups. We found that adults in the age range of 30-34 years and older individuals in the age range of 70-74 years had the highest incidence rate. Notwithstanding a minimal increase in the incidence rate of hematopoietic sarcoma in men, our results showed that the incidence rate of the disease was similar in men and women. Overall, although these findings could provide a promising perspective about the epidemiology of hematopoietic sarcoma in the Iranian population, further studies are needed to confirm the results of this study.

\section{Authors' Contribution}

HR, SS, GJ, AR, and MEA contributed to collect cases. DB and AK did statistical analysis of article. MM and HR wrote the manuscript.

\section{Conflict of Interest Disclosures}

The authors declare no conflict of interest.

\section{Ethical Statement}

The study protocol was approved by the Ethics Committee of Shahid Beheshti University of Medical Sciences and all patients' data will be retained confidentially.

\section{Funding}

This research did not receive any specific grant from funding agencies in the public, commercial, or not-for-profit sectors.

\section{Acknowledgment}

The authors thank the Shahid Beheshti University of Medical Sciences for financial support to undertake the study and the personnel of Shohadaye Tajrish Hospital and INCR in Tehran (capital of Iran) for their unwavering cooperation in performing this study.

\section{References}

1. Pileri SA, Ascani S, Cox MC, Campidelli C, Bacci F, Piccioli $\mathrm{M}$, et al. Myeloid sarcoma: clinico-pathologic, phenotypic and cytogenetic analysis of 92 adult patients. Leukemia. 2007;21(2):340-50. doi: 10.1038/sj.leu.2404491.

2. Szomor A, Baranyai F, Tornóczky T, Losonczy $H$. Penile chloroma in a patient with secondary acute myeloid leukemia. Eur J Haematol. 2002;68(5):322. doi: 10.1034/j.1600-0609.2002.02713.x.

3. Saivignesh S, Brundha MP. Myeloid sarcoma. Int J Clinicopathol Correl. 2019;3(2):41-3. doi: 10.4103/ijcpc. ijcpc_11_19.

4. Kasahara S, Tsurumi H, Hara T, Goto H, Moriwaki H. Idiopathic myelofibrosis developing isolated granulocytic sarcoma with der $(1 ; 7)(\mathrm{q} 10 ; \mathrm{p} 10)$ after splenectomy and finally transforming to acute myelogenous leukemia. Leuk Lymphoma. 2000;39(3-4):427-33. doi: 10.3109/10428190009065844.

5. Jaffe ES, Harris NL, Stein H, Vardiman JW. Pathology and genetics. In: Tumours of Haematopoietic and Lymphoid Tissues. Lyon: IARC Press; 2001.

6. Yamauchi K, Yasuda M. Comparison in treatments of nonleukemic granulocytic sarcoma: report of two cases and a review of 72 cases in the literature. Cancer. 2002;94(6):1739-46. doi: 10.1002/cncr.10399.

7. Bakst RL, Tallman MS, Douer D, Yahalom J. How I treat extramedullary acute myeloid leukemia. Blood. 2011;118(14):3785-93. doi: 10.1182/blood-2011-04-347229.

8. Ngu IW, Sinclair EC, Greenaway S, Greenberg ML. Unusual presentation of granulocytic sarcoma in the breast: a case report and review of the literature. Diagn Cytopathol. 2001;24(1):53-7. doi: $\quad$ 10.1002/1097-0339(200101)24:1<53::aiddc1009>3.0.co;2-d.

9. Antic D, Elezovic I, Milic N, Suvajdzic N, Vidovic A, Perunicic M, et al. Is there a "gold" standard treatment for patients with isolated myeloid sarcoma? Biomed Pharmacother. 2013;67(1):72-7. doi: 10.1016/j. biopha.2012.10.014.

10. Chevallier P, Mohty M, Lioure B, Michel G, Contentin N, Deconinck E, et al. Allogeneic hematopoietic stem-cell transplantation for myeloid sarcoma: a retrospective study from the SFGM-TC. J Clin Oncol. 2008;26(30):4940-3. doi: 10.1200/jco.2007.15.6315.

11. Avni B, Koren-Michowitz M. Myeloid sarcoma: current approach and therapeutic options. Ther Adv Hematol. 2011;2(5):309-16. doi: 10.1177/2040620711410774.

12. Dusenbery KE, Howells WB, Arthur DC, Alonzo T, Lee JW, Kobrinsky N, et al. Extramedullary leukemia in children with newly diagnosed acute myeloid leukemia: a report from the Children's Cancer Group. J Pediatr Hematol Oncol. 2003;25(10):760-8. doi: 10.1097/00043426-20031000000004.

13. Dores GM, Devesa SS, Curtis RE, Linet MS, Morton LM. Acute leukemia incidence and patient survival among children and adults in the United States, 2001-2007. Blood. 2012;119(1):34-43. doi: 10.1182/blood-2011-04-347872.

14. Paydas S, Zorludemir S, Ergin M. Granulocytic sarcoma: 32 cases and review of the literature. Leuk Lymphoma. 2006;47(12):2527-41. doi: 10.1080/10428190600967196.

15. Arber DA, Orazi A, Hasserjian R, Thiele J, Borowitz MJ, Le Beau MM, et al. The 2016 revision to the World Health Organization classification of myeloid neoplasms and acute leukemia. Blood. 2016;127(20):2391-405. doi: 10.1182/ blood-2016-03-643544.

16. Campidelli C, Agostinelli C, Stitson R, Pileri SA. Myeloid sarcoma: extramedullary manifestation of myeloid disorders. Am J Clin Pathol. 2009;132(3):426-37. doi: 10.1309/ajcp1za7hyzkazhs.

17. Békássy AN, Hermans J, Gorin NC, Gratwohl A. Granulocytic sarcoma after allogeneic bone marrow transplantation: a retrospective European multicenter survey. Acute and Chronic Leukemia Working Parties of the European Group for Blood and Marrow Transplantation. Bone Marrow Transplant. 1996;17(5):801-8.

18. Antic D, Elezovic I, Milic N, Suvajdzic N, Vidovic A, Perunicic M, et al. Is there a "gold" standard treatment for patients with isolated myeloid sarcoma? Biomed Pharmacother. 2013;67(1):72-7. doi: 10.1016/j. biopha.2012.10.014.

19. Tsimberidou AM, Kantarjian HM, Wen S, Keating MJ, O'Brien S, Brandt M, et al. Myeloid sarcoma is associated with superior event-free survival and overall survival compared with acute myeloid leukemia. Cancer. 2008;113(6):1370-8. doi: 10.1002/cncr.23691. 
20. Lan TY, Lin DT, Tien HF, Yang RS, Chen CY, Wu K. Prognostic factors of treatment outcomes in patients with granulocytic sarcoma. Acta Haematol. 2009;122(4):238-46. doi: 10.1159/000253592.

21. Zhang S, Charlton C, Amaraneni A, Maher K. A case of myeloid sarcoma following allogeneic HSCT presenting as localized hip pain. Case Rep Hematol. 2019;2019:2674359. doi: 10.1155/2019/2674359.

22. Zhang X, Huang P, Chen Z, Bi X, Wang Y, Wu J. Vulvar myeloid sarcoma as the presenting symptom of acute myeloid leukemia: a case report and literature review of Chinese patients, 1999-2018. Diagn Pathol. 2019;14(1):126. doi: 10.1186/s13000-019-0892-3.

23. Wang P, Li Q, Zhang L, Ji H, Zhang CZ, Wang B. A myeloid sarcoma involving the small intestine, kidneys, mesentery, and mesenteric lymph nodes: a case report and literature review. Medicine (Baltimore). 2017;96(42):e7934. doi: 10.1097/md.0000000000007934. 